Georgian Mathematical Journal

Volume 13 (2006), Number 2, 291-296

\title{
A POSITIVE ANSWER TO VELICHKO'S QUESTION
}

\author{
JINJIN LI AND ZHAOWEN LI
}

\begin{abstract}
We give positive answer to Velichko's question in which the quotient and $s$-map is replaced by a sequence-covering and $c s$-map. In addition, let $X$ have a star-countable $k$-network, then $X$ is a sequence-covering and $c s$-image of a locally separable metric space if and only if $X$ is a sequencecovering and $c s$-image of a metric space.
\end{abstract}

2000 Mathematics Subject Classification: Primary: 54E99, 54C10; Secondary: 54D55.

Key words and phrases: Sequence-covering maps, cs-mappings, cs-networks, $k$-networks, compact-countable covers, star-countable collections, cosmic spaces, $\aleph_{0}$-spaces.

\section{INTRODUCTION}

In recent years, sequence-covering maps introduced by Siwiec in [1] have again been drawing attention [2]-[5]. On the other hand, B. Qu and M. Gao introduced the concept of $c s$-map in order to study the relationships between spaces with certain compact-countable $k$-networks and certain images of metric spaces [6]. Velichko [7] posed the following interesting question about quotient and $s$-images of metric spaces: Find a $\Phi$-property such that a space $Y$ is a quotient and $s$-image of a metric and $\Phi$-space if and only if $Y$ is a $\Phi$-space which is a quotient and $s$-image of a metric space. Velichko [7] proved that a space $Y$ is a pseudo-open and $s$-image of a locally separable metric space if and only if $Y$ is a locally separable space which is a pseudo-open and $s$ image of a metric space. In this paper, it is shown that a local $\aleph_{0}$-property is a positive answer to Velichko's question if the quotient and $s$-map is replaced by a sequence-covering and $c s$-map. In addition, let $X$ have a star-countable $k$-network, then $X$ is a sequence-covering and $c s$-image of a locally separable metric space if and only if $X$ is a sequence-covering and $c s$-image of a metric space.

In this paper, all spaces are regular and $T_{1}$, all mappings are continuous and onto. $\omega=\{0\} \bigcup N$. Let us recall some basic definitions.

Definition 1.1. Let $X$ be a space, and let $\mathcal{P}$ be a cover of $X$.

(1) $\mathcal{P}$ is called compact-countable (resp. compact-finite) if for any compact subset $K$ of $X$, only countably (resp. finitely) many members of $\mathcal{P}$ intersect $K$.

(2) $\mathcal{P}$ is star-countable if for any element $P$ of $\mathcal{P}$, only countably many members of $\mathcal{P}$ intersect $P$.

(3) $\mathcal{P}$ is point-countable if for each $x \in X$, only countably many members of $\mathcal{P}$ contain $x$.

ISSN 1072-947X / \$8.00 / C) Heldermann Verlag www.heldermann.de 
(4) Let $x \in P \subset X$. $P$ is a sequential neighborhood of $x$ in $X$ [8] if whenever $\left\{x_{n}\right\}$ is a sequence converging to the point $x$, we have $\left\{x_{n}: n \geq m\right\} \subset P$ for some $m \in N$.

(5) Let $P \subset X . P$ is a sequentially open subset in $X$ [8] if $P$ is a sequential neighborhood of $x$ in $X$ for each $x \in P$. $X$ is a sequential space if each sequentially open subset in $X$ is open.

(6) $\mathcal{P}$ is a network if whenever $x \in U$ with $U$ open in $X$, we have $x \in P \subset U$ for some $P \in \mathcal{P}$. A space is a cosmic space [9] if it has a countable network.

(7) $\mathcal{P}$ is a $c s$-network [10] if whenever $\left\{x_{n}\right\}$ is a sequence converging to a point $x \in U$ with $U$ open in $X$, we have $\{x\} \bigcup\left\{x_{n}: n \geq m\right\} \subset P \subset U$ for some $m \in N$ and some $P \in \mathcal{P}$.

(8) $\mathcal{P}$ is a $k$-network for $X$ if whenever $K \subset U$ with $K$ compact and $U$ open in $X$, we have $K \subset \cup \mathcal{P}^{\prime} \subset U$ for some finite $\mathcal{P}^{\prime} \subset \mathcal{P}$; A space is an $\aleph_{0}$-space [9] if it has a countable $k$-network.

(9) $\mathcal{P}$ is an so-cover (i.e., sequentially open cover) [12] if each element of $\mathcal{P}$ is sequentially open in $X$.

Definition 1.2. Let $f: X \rightarrow Y$ be a map. Then

(1) $f$ is an $s$-map if each $f^{-1}(y)$ is separable.

(2) $f$ is a $c s$-map [6] if for each compact subset $K$ of $Y, f^{-1}(K)$ is separable.

(3) $f$ is a sequence-covering map [1] if each convergent sequence of $Y$ is the image of some convergent sequence of $X$.

(4) $f$ is a quotient map if whenever $f^{-1}(U)$ is open in $X$, we have $U$ is open in $Y$.

(5) $f$ is a pseudo-open map if whenever $f^{-1}(y) \subset V$ with $V$ open in $X$, we have $y \in \operatorname{int}(f(V))$.

Definition 1.3 ([13]). A space $X$ is sequentially separable if $X$ has a countable subset $D$ such that for each $x \in X$, there is a sequence $\left\{x_{n}\right\}$ in $D$ with $x_{n} \rightarrow x$. $D$ is called a sequentially dense subset of $X$.

Liu and Tanaka [14] showed that every cosmic space with a point-countable $c s$-network is an $\aleph_{0}$-space, the key property of which is that every cosmic space is sequentially separable.

\section{RESULTS}

Theorem 2.1. The following statements are equivalent for a space $X$ :

(1) $X$ is a sequence-covering and cs-image of a locally separable metric space.

(2) $X$ has a compact-countable cs-network consisting of cosmic subspaces.

(3) $X$ has a compact-countable cs-network, and an so-cover consisting of $\aleph_{0}$ subspaces.

(4) $X$ is a sequence-covering and cs-image of a metric space, and has an so-cover consisting of $\aleph_{0}$-subspaces.

Proof. (1) $\Rightarrow(2)$. Let $f: M \rightarrow X$ be a sequence-covering and cs-mapping, where $M$ is a locally separable metric space. Suppose $\mathcal{B}$ is a $\sigma$-locally finite 
base for $M$ consisting of separable subspaces. Put $\mathcal{P}=\{f(B): B \in \mathcal{B}\}$. Then $\mathcal{P}$ is a compact-countable $c s$-network for $X$ consisting of cosmic subspaces.

$(2) \Rightarrow(3)$. Let $\mathcal{P}$ be a compact-countable $c s$-network of $X$ consisting of cosmic subspaces. For each $P \in \mathcal{P}$, let $D(P)$ be a countable and sequentially dense subset of $P$. For each $x \in X$, put

$$
\mathcal{P}(x, 1)=\{P \in \mathcal{P}: x \in P\}, \quad D(x, 1)=\bigcup\{D(P): P \in \mathcal{P}(x, 1)\},
$$

and for each $n \geq 2$ inductively define that

$$
\begin{aligned}
& \mathcal{P}(x, n)=\{P \in \mathcal{P}: P \bigcap D(x, n-1) \neq \varnothing\}, \\
& D(x, n)=\bigcup\{D(P): P \in \mathcal{P}(x, n)\} .
\end{aligned}
$$

Let $\mathcal{P}(x)=\bigcup\{\mathcal{P}(x, n): n \in N\}$, and $U(x)=\bigcup \mathcal{P}(x)$. To complete the proof of (3), it suffices to show that $U(x)$ is sequentially open in $X$ and $\mathcal{P}(x)$ is a csnetwork for $U(x)$. If $\left\{y_{n}\right\}$ is a sequence in $X$ converging to a point $y \in U(x) \cap W$ with $\mathrm{W}$ open in $X$, then $y \in P$ for some $m \in N$ and some $P \in \mathcal{P}(x, m)$, and there is a sequence $\left\{z_{n}\right\}$ in $D(P)$ with $z_{n} \rightarrow y$, thus $\{y\} \bigcup\left\{y_{n}, z_{n}: n \geq m\right\} \subset$ $Q \subset W$ for some $m \in N$ and some $Q \in \mathcal{P}$, so $Q \in \mathcal{P}(x, m+1) \subset \mathcal{P}(x)$ and $\{y\} \bigcup\left\{y_{n}: n \geq m\right\} \subset Q \subset U(X) \cap W$. This implies that $U(x)$ is sequentially open and $\mathcal{P}(x)$ is a $c s$-network for $U(x)$.

$(3) \Rightarrow(1)$. First, we shall show that $X$ has a compact-countable $c s$-network $\mathcal{P}$ consisting of $\aleph_{0}$-subspaces. Let $\mathcal{P}^{\prime}$ be a compact-countable $c s$-network of $X$ which is closed under finite intersections, and let $\mathcal{U}$ be an so-cover of $X$ consisting of $\aleph_{0}$-subspaces. Put

$$
\mathcal{P}=\left\{P \in \mathcal{P}^{\prime}: P \subset U \text { for some } U \in \mathcal{U}\right\} .
$$

Then $\mathcal{P}$ is still a $c s$-network for $X$. Indeed, let $x \in W$ with $W$ open in $X$. If $\left\{x_{n}\right\}$ is a sequence converging to the point $x \in X$, put

$$
\begin{gathered}
\mathcal{P}_{x}^{\prime}=\left\{P \in \mathcal{P}^{\prime}: x \in P \subset W \text { and } P \text { contains all but finite many } x_{n}\right\} \\
=\left\{P_{n}: n \in N\right\} .
\end{gathered}
$$

For each $n \in N$, take $Q_{n}=\bigcap_{i \leq n} P_{i}$, then $Q_{n} \in \mathcal{P}_{x}^{\prime}$. Let $U_{x} \in \mathcal{U}$ be a sequential neighborhood of $x$ in $X$. If there is $q_{n} \in Q_{n} \backslash U_{x}$ for each $n \in N$, and $\mathrm{G}$ is open in $X$ with $x \in G$, then $P_{k} \subset G$ for some $k \in N$ because $\mathcal{P}^{\prime}$ is a cs-network for $X$, thus $q_{n} \in Q_{n} \subset P_{k} \subset G$ when $n \geq k$, and $q_{n} \rightarrow x$, a contradiction. Hence $Q_{m} \subset U_{x}$ for some $m \in N$, and $Q_{m} \in \mathcal{P}$. Therefore $\mathcal{P}$ is a compact-countable $c s$-network for $X$ consisting of $\aleph_{0}$-subspaces. Let $\mathcal{P}=\left\{P_{\alpha}: \alpha \in \bigwedge\right\}$. For each $\alpha \in \bigwedge$, by Theorem in [9], there are a separable metric space $M_{\alpha}$ and a sequence-covering $f_{\alpha}: M_{\alpha} \rightarrow P_{\alpha}$. Put $M=\bigoplus_{\alpha \in \Lambda} M_{\alpha}, Z=\bigoplus_{\alpha \in \Lambda} P_{\alpha}$ and $f=\bigoplus_{\alpha \in \Lambda} f_{\alpha}: M \rightarrow Z$. Then $M$ is a locally separable metric space and $f$ is a sequence-covering map. Assume $h: Z \rightarrow X$ to be a natural map, and let $g=h \circ f: M \rightarrow X$. Then $g$ is a sequence-covering $c s$-mapping.

$(3) \Leftrightarrow(4)$. It suffices to show that $X$ has a compact-countable cs-network if and only if $X$ is a sequence-covering and $c s$-image of a metric space. Let $X$ 
be a space with a compact-countable $c s$-network $\mathcal{P}$. We can suppose that $\mathcal{P}$ is closed under finite intersections. Denote $\mathcal{P}$ by $\left\{P_{\alpha}: \alpha \in A\right\}$. Let $A_{i}$ denote the set $A$ with a discrete topology for each $i \in N$. Put

$$
M=\left\{\beta=\left(\alpha_{i}\right) \in \prod_{i \in N} A_{i}:\left\{P_{\alpha_{i}}: i \in N\right\} \text { is a network at some point } x(\beta) \text { in } X\right\},
$$

then $M$ is a metric space, and $f: M \rightarrow X$ defined by $f(\beta)=x(\beta)$ is a $c s$ map. We shall show that $f$ is sequence-covering. For a sequence $\left\{x_{n}\right\}$ of $X$ converging to a point $x_{0}$ in $X$, we can assume that all $x_{n}$ 's are distinct. Let $K=\left\{x_{m}: m \in \omega\right\}$, and let $K \subset U$ with $U$ open in $X$. A subset $\mathcal{F}$ of $\mathcal{P}$ is said to have the property $F(K, U)$ if $\mathcal{F}$ satisfies the following conditions:

(1) $\mathcal{F}$ is finite;

(2) $\varnothing \neq P \bigcap K \subset P \subset U$ for each $P \in \mathcal{F}$;

(3) for each $x \in K$ there is a unique $P_{x} \in \mathcal{F}$ with $x \in P_{x}$;

(4) if $x_{0} \in P \in \mathcal{F}$, then $K \backslash P$ is finite.

Put

$$
\{\mathcal{F} \subset \mathcal{P}: \mathcal{F} \text { has the property } F(K, X)\}=\left\{\mathcal{F}_{i}: i \in N\right\}
$$

For each $i \in N$ and each $m \in \omega$, there is $\alpha_{i m} \in A_{i}$ with $x_{m} \in P_{\alpha_{i m}} \in \mathcal{F}_{i}$. It can be checked that $\left\{P_{\alpha_{i m}}: i \in N\right\}$ is a network at the point $x_{m}$. Let $\beta_{m}=\left(\alpha_{i m}\right)$ for each $m \in \omega$, then $\beta_{m} \in M$ and $f\left(\beta_{m}\right)=x_{m}$. For each $i \in N$, there is $n(i) \in N$ such that $\alpha_{i n}=\alpha_{i 0}$ if $n \geq n(i)$. Thus the sequence $\left\{\alpha_{i n}\right\}$ converges to $\alpha_{i 0}$ in $A_{i}$, and the sequence $\left\{\beta_{n}\right\}$ converges to $\beta_{0}$ in $M$. This shows that $f$ is a sequence-covering map.

Conversely, suppose that $f: M \rightarrow X$ is a sequence-covering and cs-map, where $\mathrm{M}$ is a metric space. Let $\mathcal{B}$ be a $\sigma$-locally-finite base for $\mathrm{M}$, then $\{f(B)$ : $B \in \mathcal{B}\}$ is a compact-countable $c s$-network for $X$.

Corollary 2.2. The following statements are equivalent for a space $X$ :

(1) $X$ is a sequence-covering and quotient cs-image of a locally separable metric space.

(2) $X$ is a local $\aleph_{0}$-space and a sequence-covering, and a quotient cs-image of a metric space.

(3) $X$ is a sequential and local $\aleph_{0}$-space with a compact-countable cs-network.

Theorem 2.3. Let $X$ have a star-countable k-network. Then $X$ is a sequencecovering and cs-image of a locally separable metric space if and only if $X$ is a sequence-covering and cs-image of a metric space.

Proof. We prove only the "if" part. We have that a space $X$ is a sequencecovering and $c s$-image of a metric space if and only if $X$ has a compact-countable $c s$-network from the proof of Theorem 2.1. Let $\mathcal{P}$ be a compact-countable $c s$ network for $X$, here we can assume that $\mathcal{P}$ is closed under finite intersections. Since $X$ has a star-countable $k$-network $\mathcal{R}$, in view of Lemma 1.1 in [15], $X$ is the disjoint union of $\left\{X_{\alpha}: \alpha \in \bigwedge\right\}$ satisfying the following conditions:

(a) each $X_{\alpha}$ is an $\aleph_{0}$-space which is the countable union of elements of $\mathcal{R}$.

(b) $\left\{X_{\alpha}: \alpha \in \bigwedge\right\}$ is compact-finite. 
Let $\mathcal{P}^{\prime}=\left\{P \in \mathcal{P}: P\right.$ is an $\aleph_{0}$-space of $\left.X\right\}$. Then $\mathcal{P}^{\prime}$ is a $c s$-network for $X$. Indeed, let $L=\left\{x_{n}: n \in N\right\}$ converge to $x$ with $x \in U$ and $U$ is open. Let $\mathcal{P}_{x}=\{P \in \mathcal{P}: x \in P, P \subset U$ and $L$ is eventually in $P\}$. Then $\mathcal{P}_{x}$ is countable. We take a subfamily $\mathcal{P}_{x}^{\prime}=\left\{P_{n}: n \in N\right\}$ of $\mathcal{P}_{x}$ such that $P_{n} \supset P_{n+1}$ for any $n \in N$ and $\mathcal{P}_{x}^{\prime}$ is a network of $x$ in $X$. If $\mathcal{P}_{x}^{\prime} \cap \mathcal{P}^{\prime}=\varnothing$, this means that each $P_{n}$ meets uncountably many $X_{\alpha}$. Pick $z_{n} \in P_{n} \bigcap X_{\alpha(n)}$ for some $\alpha(n) \in \bigwedge$, where $X_{\alpha}(n) \neq X_{\alpha}(m)$ if $n \neq m$. Then $\left\{z_{n}: n \in N\right\}$ is a convergent sequence with $z_{n} \rightarrow x$. The compact set $\{x\} \bigcup\left\{z_{n}: n \in N\right\}$ meets infinitely many $X_{\alpha}$. This is a contradiction to (b). Thus $\mathcal{P}^{\prime}$ is a compact-countable $c s$-network for $X$ consisting of $\aleph_{0}$-spaces. By Theorem $2.1, X$ is a sequence-covering and $c s$-image of a locally separable metric space.

\section{ACKNOWLEDGEMENT}

This work is supported by the NSF of China.

\section{REFERENCES}

1. F. SiwiEc, Sequence-covering and countably bi-quotient mappings. General Topology and Appl. 1(1971), No. 2, 143-154.

2. Shou Lin, Sequence-covering s-mappings. (Chinese) Adv. in Math. (China) 25(1996), No. 6, 548-551.

3. Y. TAnakA and ShengXiang Xia, Certain $s$-images of locally separable metric spaces. Questions Answers Gen. Topology 14(1996), No. 2, 217-231.

4. Li Zhen Zhou, Some sequence-covering s-images of locally separable metric spaces. (Chinese) Acta Math. Sinica 42(1999), No. 4, 577-582.

5. Y. Tanaka, Metrization. II. Topics in general topology, 275-314, North-Holland Math. Library, 41, North-Holland, Amsterdam, 1989.

6. Zhi Bin QU and Zhi Min GaO, Spaces with compact-countable $k$-networks. Math. Japon. 49(1999), No. 2, 199-205.

7. N. V. Velichko, Quotient spaces of metrizable spaces. (Russian) Sibirsk. Mat. Zh. 28(1987), No. 4, 73-81, 225.

8. S. P. Franklin, Spaces in which sequences suffice. Fund. Math. 57(1965), 107-115.

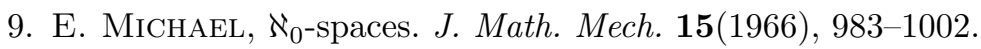

10. J. A. Guthrie, A characterization of $\aleph_{0}$-spaces. General Topology and Appl. 1(1971), No. 2, 105-110.

11. P. O'Meara, On paracompactness in function spaces with the compact-open topology. Proc. Amer. Math. Soc. 29(1971), 183-189.

12. Shou Lin and Penfei Yan, On the sequence-covering and compact images of metric spaces. To appear.

13. JING XIAN Sun, Generalizations of the principle of ordered sets in nonlinear functional analysis. (Chinese) J. Systems Sci. Math. Sci. 10(1990), No. 3, 228-232.

14. ChuAn Liu and Y. TANakA, Spaces having $\sigma$-compact-finite $k$-networks, and related matters. Topology Proc. 21(1996), 173-200.

15. Y. IKedA and Y. TANAKA, Spaces having star-countable $k$-networks. Topology Proc. 18(1993), 107-132. 
(Received 4.01.2005)

Author's addresses:

Jinjin Li

Dept. of Math.

Zhangzhou Teachers' College

Zhangzhou, Fujian 363000

P.R. China

E-mail: jinjinli@fjzs.edu.cn

Zhaowen $\mathrm{Li}$

Dept. of Math.

Changsha University of Electric Power

Changsha, Hunan 410077

P.R. China 